\title{
Multidisciplinary Integrated Knowledge Modeling of Complex Product Design
}

\author{
http://dx.doi.org/10.3991/ijoe.v9iS4.2645 \\ Haiqiang Liu, Ming Lv \\ Hangzhou Dianzi University, Hangzhou, China
}

\begin{abstract}
Based on analysis of application characteristics of product design knowledge, the design process of complex product was presented. A framework structure of product integrated design knowledge model for supporting multidisciplinary design was proposed, the fusion method of product integrated design knowledge was also explored, and design knowledge acquisition of multidisciplinary design along with evaluating satisfaction degree in process and method of objection were analyzed. Associated with the methodology of multidisciplinary design optimization, a prototype system supporting multidisciplinary design was developed based on ideal framework of product integrated design knowledge model with the software development tool Genexus.
\end{abstract}

Index Terms-Design Process, Multidisciplinary Design, Knowledge Model, Satisfaction Degree

\section{INTRODUCTION}

With increasingly severe product marketing competition, product development and design all have changed a great deal in process and pattern. The modern industrial enterprises adopted many advanced methods and supporting technologies for less cost, faster, and higher performance. meanwhile, object of engineering design be also more and more complicated, such as design of complex product, usually need to multiple disciplinary to be in collaborative development and design. Complex product refers to a product of complicated structure, technology intensive, high with manufacturing request and cost, complex process management, and diversified requirements from customers[1-2].There are two remarkable characteristics of product design: the first one is knowledge density, turn purely geometrical optimization design into performance design and variant design based on knowledge; the second one is complexity of multidisciplinary collaboration, for design of complex mechanical product, usually have various function and performance requirements.

Considering that design of mechanical product is a process which is a complicated, multidisciplinary collaborative and weak theoretical supporting, much design knowledge and experience can hardly express in rule, so case-based reasoning method is gaining extensive attention and application[3]. In this paper, the design process of multidisciplinary design optimization was analyzed, product integrated design knowledge model for supporting multidisciplinary design was proposed, integrating product related design knowledge with design instance and rule synthesis and providing necessary design knowledge for designers.

\section{DESIGN Process OF COMPLEX Product}

Multidisciplinary design is a field of engineering that use optimization methods to solve design problems incorporating a number of discipline , it can be defined as a formal methodology for the design of complex coupled systems in which the synergistic effects of coupling between various interacting disciplines are explored and exploited at every stage of the design process.

The design of modern complex product is a multidisciplinary problem, which including multidisciplinary analysis, multidisciplinary design and multidisciplinary optimization, usually involve design data coupling of multiple disciplinary. Design process of complex product is a collaborative work pattern of multi disciplinary, each design subtask usually isn't sequential or concurrent, and has multi directions and different capacity levels of data exchange. Design process is classified into 3 patterns according to related way of subtask[4], As fig.1, multidisciplinary design process of complex product usually is hybrid pattern.

Fig.2 is regular design process and related design knowledge of complex product. The left side of Fig. 2 is regular design process, mainly includes conceptual design, preliminary design and detail design, involves design evaluation in different stages[5]. The right side of Fig.2 is related design knowledge of complex product, there are mainly comprehensive knowledge of design task decomposition, design specifications, design criterion, design experience, process knowledge, multidisciplinary knowledge, design modification knowledge and design check knowledge, etc.

\section{INTEGRATED DESIGN KNOWLEDGE MODELING}

The product design involves to multidisciplinary, is an activity of knowledge density. Designers generally decomposed product design task into is a series of simple subtask by methods of decomposition synthesis. Product design, especially for variant design, can reuse design experience and design knowledge by design practical accumulation to adapt to fast changing market need[6]. Therefore, it is urgently need to study design knowledge model, in order to provide related design knowledge for designers, and help designers to make a reasonable decision based on successful design case or design mistake in product design process.

Product integrated design knowledge model is based on product data model, associating description knowledge of product design, creating integrated expression of design instance and rule, and obtaining comprehensive optimal 


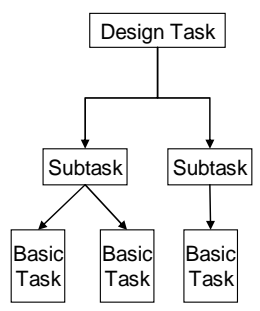

(1) Top-down Pattern
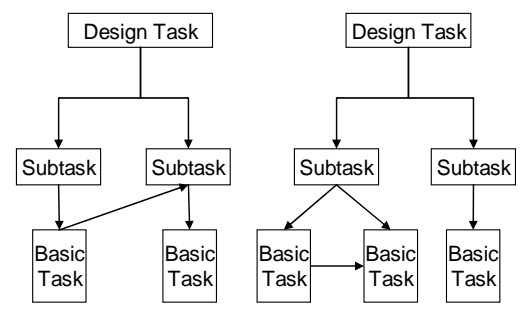

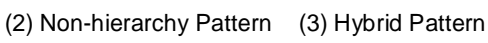

Figure 1. Pattern of design process

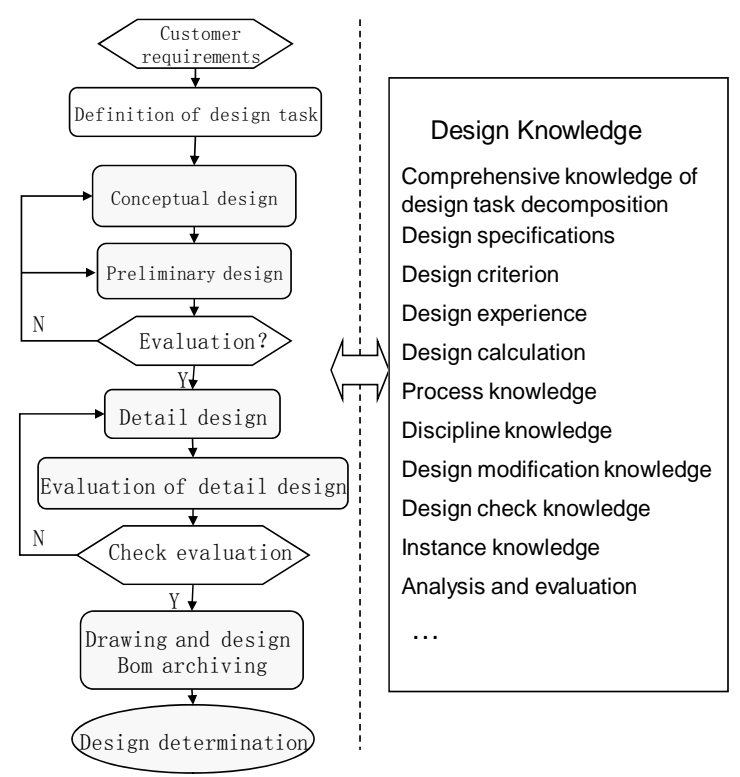

Figure 2. Regular design process and related design knowledge of complex product

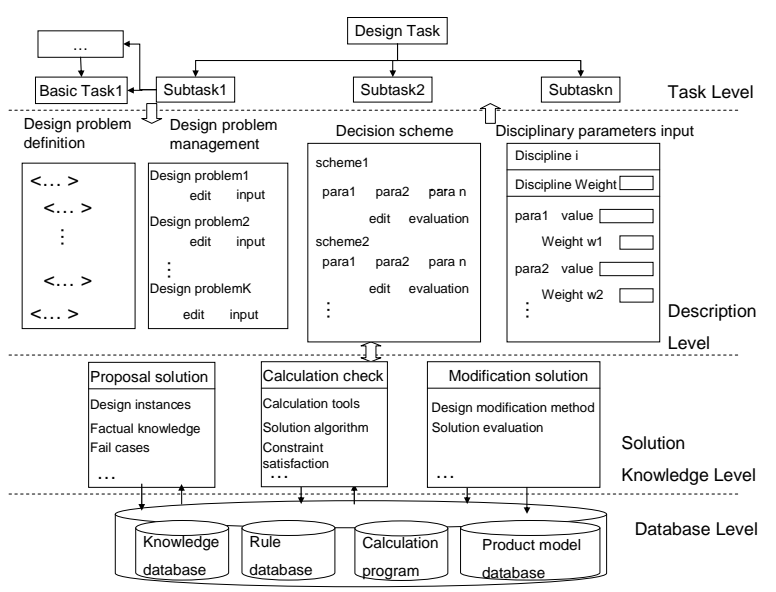

Figure 3. Product integrated design knowledge model

design solution. Product integrated design knowledge model, as shown in Fig.3. The first level is task level,namely decomposition structure of unified product design task. The second level is description level, description of design problems based on selected design task node, includes four steps of design problem definition, design problem management, decision scheme management and discipline parameters input. The third level is solution knowledge level, solution of specific task, realizing specified representation of solution knowledge according to structure definition of task template. The fourth level is database level, includes knowledge database, rule database, calculation database, product model database and interface module, used to support solution knowledge level. Each level of model are mutually related through mapping management, subtask and basic task of task level are mapped and decomposed in description level, methods of description are mapped to knowledge of solution knowledge level. That can effectively Layered and classified for design knowledge through hierarchy division of knowledge model, thus realizing on the maximization of reusing knowledge.

Function of design problem definition is mainly support to participant can expediently edit description information of design problem to meet a system requirements. Function of design problem management is carried on a total management towards information of design problem, include operations of design information, such as search, read, modify, add, delete etc. Function of decision scheme management is mainly to build decision tree according to the selected design problems, and carry on operations to related decision set information in the database, such as access and modification. Function of disciplinary parameters input is mainly to provide parameters, weight of disciplinary for decision scheme, and execute the corresponding access operation to integrated database.

\section{FUSION OF INTEGRATED DESIGN KNOWLEDGE}

Geometric description is essential in product design process, inevitably involved the fusion design knowledge with geometric model, and design knowledge of complex product is lack of criterion, weak of cross, and hard to express by single knowledge expression. In this paper, the knowledge of product design was expressed in integrated expression of design instance and rule, key technologies including: knowledge expression of product design, knowledge acquisition of multidisciplinary design and evaluation of satisfaction degree.

\section{A. Knowledge Expression of Design}

Design problem is expressed with document of XML format, information of XML format has a tree structure, after creating the decision tree of XML format, the hierarchical relation of various design item to be embodied. Traversing design problem by XML Paser, and then obtained all decisions about design problem by recording traversal paths, finally keep these information all in decision table of integrated database. Taking design tumbler stage of rotor for example, expression of design knowledge problem is mean as follows:

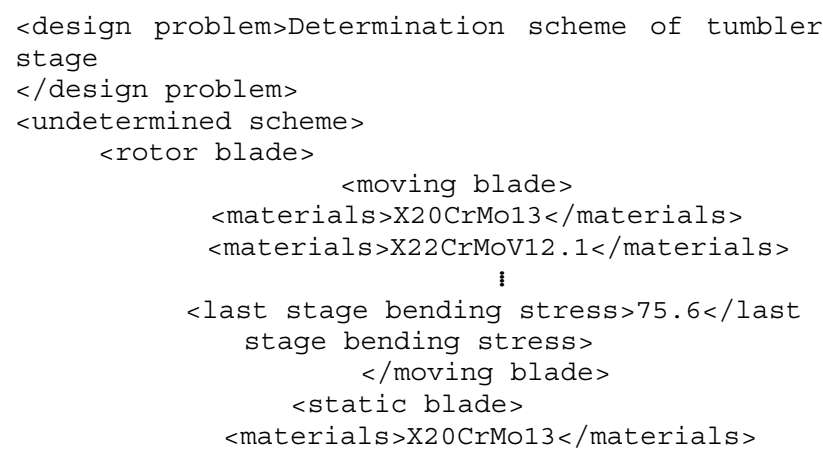




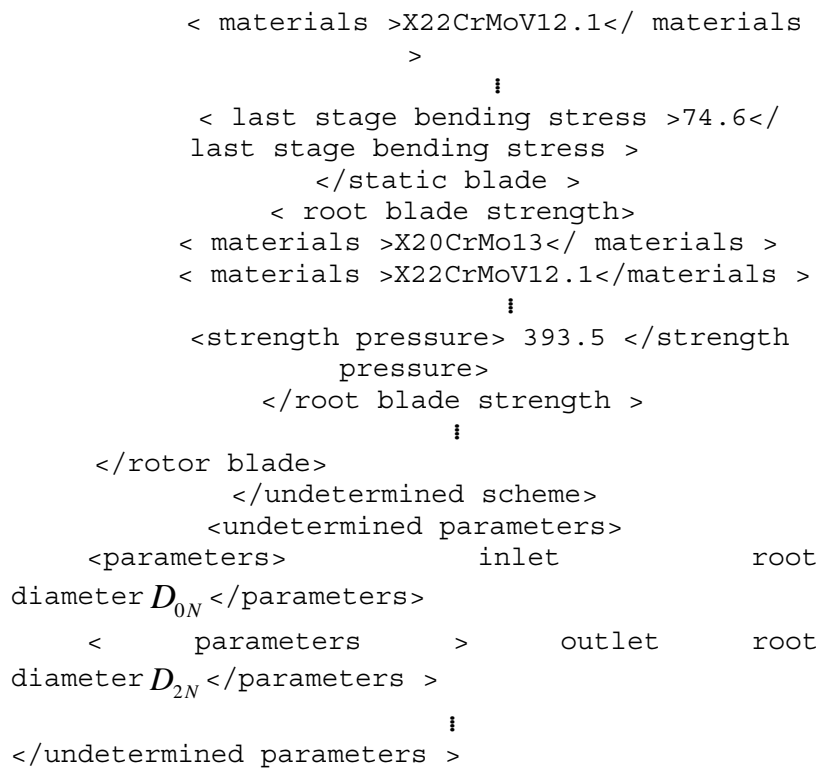

\section{B. Evaluation of Satisfaction Degree}

In the process of product multidisciplinary design optimization, different discipline pursuing of different objective, designer of different discipline uses specialized knowledge, design rules and experience to design a product, obtain maximum satisfy solution to corresponding discipline. Multidisciplinary design is a multi-objective decision problem, which of solution should be Pareto not-dominated solution set, each discipline can't acquire the superior value of objective in the meantime, but can arrive at certain satisfaction degree of solution.

Because satisfaction degree is always descending when make a concession to some discipline, the first derivative of concession is negative in satisfaction function of discipline. The second derivative of concession is less than or equal to 0 , as each discipline always attempts to make objective of oneself as far as possible optimum. Normalized treatment of concession $C$ and satisfaction degree $S$, that is $0 \leq C \leq 1$ and $0 \leq S \leq 1$.

Satisfaction degree function of discipline to design problem $j$, as is follows:

$$
\begin{aligned}
& S_{j}^{\prime}\left(C_{j}\right)=1-C_{j}^{x} \\
& S_{j}^{\prime \prime}\left(C_{j}\right)=S_{j}^{\prime}\left(1-C_{j}\right)=1-\left(1-C_{j}\right)^{x}
\end{aligned}
$$

Designer can express his design intention and strategy by selecting $\mathrm{x}$ value $(x \geq 1)$ and determine the satisfaction degree function. Comprehensive satisfaction function of discipline to design problem $j$, as is follows:

$$
S_{j}\left(C_{j}\right)=\sum_{i 1=1}^{n 1} W_{d}^{i 1} W_{j}^{i 1} S_{j}^{\prime}\left(C_{j}\right)+\sum_{i 2=1}^{n 2} W_{d}^{i 2} W_{j}^{i 2} S_{j}^{\prime \prime}\left(C_{j}\right)
$$

For $n 1, n 2$ denote, respectively, the number of discipline, comprehensive satisfaction degree $S_{j}$ is determined by formula (3), the maximum concession $C_{j}^{*}$. And then designer can obtain solution to corresponding design problem $j$ through mapping in the physical domain.

\section{Application}

Based on previous product integrated design knowledge model and fusion method of product integrated design knowledge, the author developed a supporting multidisciplinary design optimization prototype system by the software tools named Genexus. Design example of flowpath of some steam turbine rotor was researched, which involving these disciplines about structure design, thermodynamic design and strength design.

As follows that is implementing process of design based on product integrated design knowledge model, which divided into four steps:

Step 1 Product designer need to register and login in system, after the pass of system authorization can carry on to input, query and modify information of design

Step 2 Work project of establishment, establish design task of design project, the whole system takes design task as basic unit and each task is a independent analytical and optimizing process to corresponding discipline.

Step 3 Fill in acquisition table of product integrated design knowledge, according to the structured expression of design knowledge in product design knowledge model, design knowledge with specific design task are associated, describing design problem of task node, include design problem definition, design problem management, decision scheme management and discipline parameters input.

Step 4 Calling the related design knowledge in solution knowledge level of product integrated design knowledge model, constraint satisfying condition and calculation programs to execute design process, as shown in Fig.4. Finally carry on analysis and evaluation of satisfaction degree to design objective.

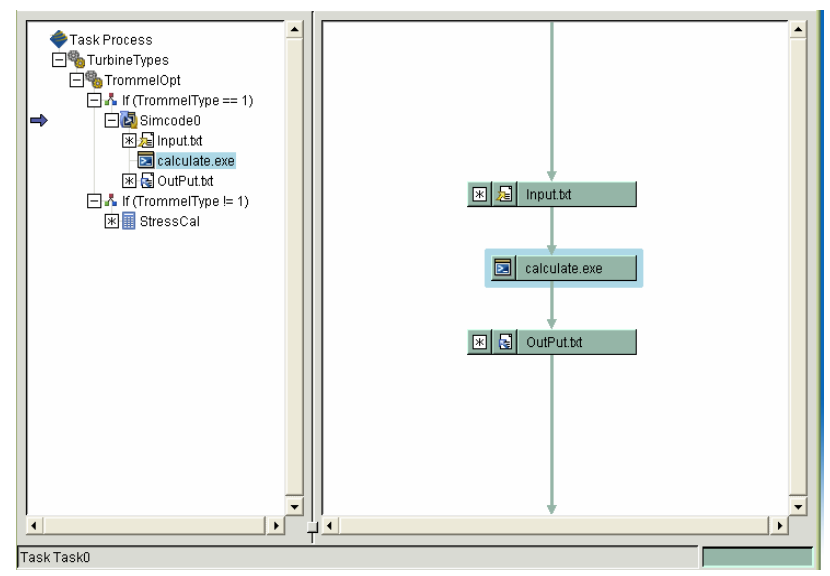

Figure 4. Execution of design task and integrated modules

Four steps are a cycling constantly process, system can continuously enrich design knowledge and experience according to design need. Make an improvement and optimization to the knowledge model, carry on a modification to product data model, transfer changed information to system database, system will update product integrated design knowledge model and obtain the evaluation solution of design product.

Each discipline validates function pattern of its satisfaction based on design intention, according to formula (1) and (2), structure design $x=3.0$, thermodynamic design discipline $x=2.0$ and strength design $x=2.5$, the comprehensive satisfaction degree 


\section{MultidisciplinARy INTEGRATEd KNOWLEdGE MODELING OF COMPLEX ProduCt Design}

can be achieved by formula (3) when $w_{1}=0.4, w_{2}=0.3, w_{3}=0.3$, thus satisfaction degree curves of objective can be drawn in Fig.5.

\section{CONCLUSIONS}

The product integrated design knowledge model is suitable for supporting multidisciplinary design optimization. On the basis of knowledge expression of product design, knowledge acquisition of multidisciplinary design and evaluation of satisfaction degree, realizing on Fusion of product integrated design knowledge. Product integrated design knowledge model can provide a framework structure for design process of complex product multidisciplinary design optimization and that can effectively Layered and classified for design knowledge through hierarchy division of knowledge model, thus realizing on the maximization of reusing knowledge.

In the next research step, a further improvement will be made to description method of design knowledge and prototype system for supporting complex product process model.

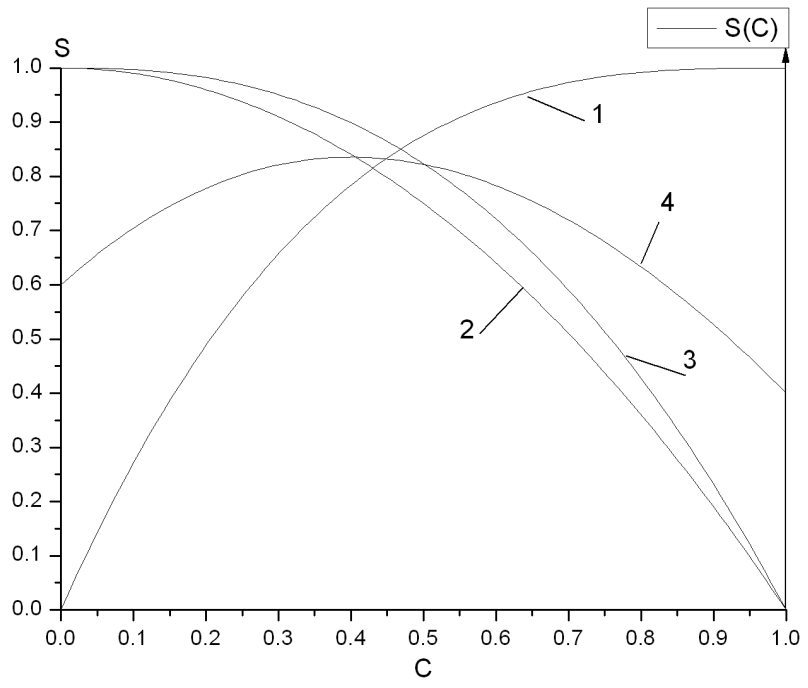

Figure 5. Satisfaction degree curves of objective

\section{ACKNOWLEDGMENT}

This research was supported in part by Key Discipline of The Ocean Mechatronic Equipments Technology, Zhejiang Provincial Natural Science Foundation of China (Grant No. LQ12E05002) and National Natural Science Foundation of China (Grant NO. 51209062 )

\section{REFERENCES}

[1] Li Bo-hu,Chai Xu-dong. "Virtual Prototyping Engineering of Complex Product,” Computer Integrated Manufacturing Systems, Vol.8, pp. 678-683, 2003.

[2] Szykman S, Sriram RD. "Design Repositories: Engineering Design's New Know ledge Base,” Intelligent Systems, IEEE, Vol.3, pp. 47-57, 2000.

[3] Shi Qi, Du Jiang, Li Yuan, et al. “ACBR (Case-Based Reasoning) Conceptual Design Model for Product Development,” Journal of Northwestern Polytechnical university, Vol.20, pp. 203-207,2002.

[4] Liao Xing, Wang Zhen-hua. "Design Process Modeling and Reconstructing Technology in Multidisciplinary Design Optimization," Aeronautical Manufacturing Technology, Vol.14, pp. 87-88, 2007.

[5] Wang Ke, Liao Wen-he, Guo Yu, et al. "Study on Modeling of the Product Design Knowledge Based on Task-structure,” China Mechanical Engineering, Vol. 19, pp. 308-310, 2008.

[6] Chapman C B, Pinfold M. "Design Engineering: a Need to Rethink the Solution Using Knowledge Based Engineering," Knowledge-based System, Vol. 12, pp. 257-267, 1999. http://dx.doi.org/10.1016/S0950-7051(99)00013-1

\section{AUTHORS}

Liu Haiqiang is now with the Department of Mechanical Engineering Hangzhou Dianzi University, Hangzhou, CO 310018 CHINA (e-mail: Liuhaiqiang1980@126.com).

Lv Ming is now with the Department of Mechanical Engineering Hangzhou Dianzi University, Hangzhou, CO 310018 CHINA (e-mail: Lvmingcn@163.com).

This article is an extended and modified version of a paper presented at the International Conference on Mechanical Engineering, Automation and Material Science (MEAMS2012), held 22-23 December 2012, Wuhan, China. This work was supported in part by Zhejiang Provincial Natural Science Foundation of China (Grant No. LQ12E05002) and National Natural Science Foundation of China (Grant NO. 51209062 ) Received 30 March 2013. Published as resubmitted by the authors 01 May 2013. 